Proceedings of SALT 26: 350-370, 2016

\title{
Counting in context: Count/mass variation and restrictions on coercion in collective artifact nouns *
}

\author{
Peter R. Sutton \\ Hana Filip \\ Heinrich Heine University Düsseldorf Heinrich Heine University Düsseldorf
}

\begin{abstract}
A major factor grounding the mass/count distinction is the (non-)resolution of overlap in context. We argue that counting presupposes that nouns be interpreted relative to counting contexts, which are contexts enforcing a resolution of overlap in noun denotations. While, in this respect, we largely follow some suggestions in Rothstein 2010 and Landman 2011, what is novel about our proposal is the role of context in the (non-)resolution of overlap. Lexical entries of mass nouns specify the null context as the context for evaluation, which makes them uncountable. The reason for this is that the null context allows for overlap in noun denotations, because it is the union of the interpretations of the predicate at all counting contexts. In contrast, lexical entries of count nouns do not specify such a context, and therefore their counting context may vary from utterance to utterance. Adopting this semantics has two major benefits. First, we can predict, on semantic grounds, for a large class of nouns, when we can(not) expect to find mass/count variation cross- and intralinguistically. Second, we are able to explain why mass 'collective artifact' nouns (aka 'object' or 'fake' mass nouns) resist mass-to-count coercion.
\end{abstract}

Keywords: mass, count, mereology, context, coercion

\section{Outline}

So-called 'object' or 'fake' mass nouns like furniture, footwear, and kitchenware in English have posed major challenges to some theories of the mass/count distinction (see e.g., Chierchia 2010), but also served as one of the main data points for the development of others (see e.g., Chierchia 1998; Landman 2011). In this paper, we will address two of their puzzling properties. First, in contrast to prototypical mass nouns like water, furniture-like mass nouns exhibit a striking variation in count/mass lexicalization patterns in natural languages. Second, when lexicalized as mass nouns, they show stubborn resistance to mass-to-count coercion. The goal of this paper is

* We thank Todor Koev for comments and help with Bulgarian examples, Natalia Beckmann for providing us with German corpus data, Max Gottwein for help with our German examples, our Finnish consultants Jouni Kahila, Sami Kumpula and Esa Lukkarinen, and attendees of SALT 26. This research was funded by the German Research Foundation (DFG) CRC991, project C09.

(C)2016 Sutton \& Filip 
to argue that both these puzzling properties can be motivated with recourse to how counting contexts are manipulated in lexical entries.

The outline of this paper is as follows. In Section 2, we lay out the relevant data. In Section 3, we outline the contemporary accounts of the mass/count distinction that have most influenced our current proposal, namely, Rothstein 2010 and Landman 2011. In Section 4, we motivate a synthesis of Rothstein and Landman that yields better coverage of count/mass variation. In Section 5, building on this synthesized account, we show that our analysis of the mass/count distinction correctly predicts that there should be a great deal of variation in mass or count lexicalization of 'object' mass nouns and far more stability in count and mass lexicalization of prototypical cases of mass and count nouns at the polar ends of the count/mass continuum.

In Section 6, we outline a simplified semantics for container-classifier readings of nouns (e.g. bowl (of)), and for unit-extracting classifiers (e.g. item (of)). We distinguish between mass-to-count coercion for portion readings, and sentences where classifiers are provided explicitly: namely, explicit classifiers supply their own contexts, implicitly supplied classifier concepts do not. As we then show, this proposal, combined with our semantics from Section 5, yields the correct constraints on mass-to-count coercion. In Section 7, we address the outstanding question of count/mass variation in 'granular' nouns such as rice and lentils.

\section{Data}

\subsection{Cross-linguistic count/mass variation}

One of the most robust tests, in number marking languages at least, for the countability status of a noun is a felicitous use in direct modification NP constructions with a numerical expression, possibly also modulo linguistic and extra-linguistic context. This test is, for example, applicable in both English and Finnish: (1a) and (1b) are both felicitous, indicating that chair and tuoli ('chair', Finnish) are count nouns, whereas, barring coerced readings, (2a) and (2b) are both highly infelicitous indicating that mud and muta ('mud', Finnish) are both mass nouns.

(1) a. I bought two chairs.

b. Ost-i-n kaksi tuoli-a.

buy-PAST-1SG two chair.SG-PART

'I bought two chairs.'

(2) a. ?? I found two mud(s) on the floor.

b. ?? Löys-i-n kaksi muta-a lattia-lta.

find-PAST-1SG two mud.SG-PART floor.SG-ABL

'I found two mud(s) on the floor.' 
Furthermore, such prototypical cases show a markedly strong tendency to be lexicalized as count and mass, respectively. However, as has long been observed (since at least Quine 1960), there is a class of nouns in English, variously described as fake mass, object mass, superordinate mass nouns, that have count counterparts in a number of other languages. Given that we would like a label to group these nouns together, the 'mass' in labels such as fake mass would be a misnomer. For example, the English mass noun footwear has a plural count counterpart in Finnish, jalkine-et ('footwear-PL'). Hence, we label nouns in this class as collective artifacts ('artifacts', given that they tend to denote man-made objects manufactured to fulfill special functions). Some examples are given below. While furniture, footwear, and jewelry in English are infelicitous when pluralized, as the infelicity of (3a), (4a), and (5a), shows, the corresponding nouns in Finnish can be pluralized and occur in count syntax, and hence are count, as the felicity of (3b), (4b) and (5b) indicates:

(3) a. ?? I bought three furnitures: a table and two chairs.

b. Ost-i-n kolme huonekalu-a: pöydä-n ja kaksi buy-PAST-1SG three furniture.SG-PART table.SG-ACC and two tuoli-a. chair.SG-PART

'I bought three items/pieces of furniture: a table and two chairs'

a. ?? I took two footwear(s) from the shelf.

b. Ot-i-n kaksi jalkine-tta hylly-ltä. take-past-1SG two footwear.SG-PART shelf.SG-ABL 'I took two pieces/items of footwear from the shelf'

(5) a. ?? I'm looking for two gold jewelry/jewelries.

b. Etsi-n kah-ta kultais-ta koru-a. look.for-1SG two-PART gold.SG-PART jewelry.SG-PART

'I'm looking for two pieces/items of gold jewelry'

Count/mass variation patterns require an explanation. While there are classes of nouns that are stably lexicalized as count (chair) or mass ( $m u d$ ) cross-linguistically, there are other classes of nouns that exhibit variation in count/mass lexicalization patterns. This prominently includes collective artifact nouns. It is the goal of this paper to explain these facts. Towards this goal we will propose a novel semantic model of countability that is, in part, inspired by Rothstein 2010 and Landman 2011.

\subsection{The puzzle of collective artifacts: Restrictions on mass-to-count coercion}

Collective artifacts exhibit further puzzles. They strongly resist mass-to-count coercion, for both 'apportioning' (6) and kind interpretations (7). This is well- 
Counting in context

known, but rarely mentioned, with the notable exception of Rothstein (2015). ${ }^{1}$

?? Can you bring three furnitures to our office, please?

Int: 'Can you bring three items of furniture to our office, please?'

(7) ?? I ordered three furnitures: chairs, tables and cabinets/kitchen, living room, and office.

Int: 'I ordered three kinds of furniture...'

In contrast, prototypical mass nouns allow coercion into packaged portions and kind interpretations much more freely. It has been well documented, since at least Pelletier 1975, that directly attaching numerical expressions to mass nouns can give rise to packaging coercions, provided the context sanctions them. This is very easy for some nouns in conventionalized portions, as in (8), less easy for others, as in (9), but perfectly possible even for non-conventionalized portions, as in (10).

(8) Three waters, please!

e.g. Three [GLASSES/BOTTLES OF] water.

(9) Context: Waiter talking to kitchen

Table 12 want two more (basmati) rices.

e.g. two more [BOWLS OF] (basmati) rice.

(10) Context: Yield points of three different mud samples before contamination.

The three muds experienced particles dispersion at the same temperature with different yield points. ${ }^{2}$

(i) The three [SAMPLES OF] mud..., (ii) The three [KINDS OF] mud... .

Coerced (sub)kind interpretations of prototypical mass nouns are illustrated below with water in (11), rice in (12), and mud in (10).

(11) I got three waters for the party: still, sparkling, and fruit-flavored for the kids.

(12) Context: Three kinds of rice, Calmati, Texmati, Kasmati.

These three rices have basmati's viscosity and cooking style, but smaller individual grains. ${ }^{3}$

1 In German, packaging coercion for mass collective artifacts such as Besteck ('cutlery') is possible:

(i)

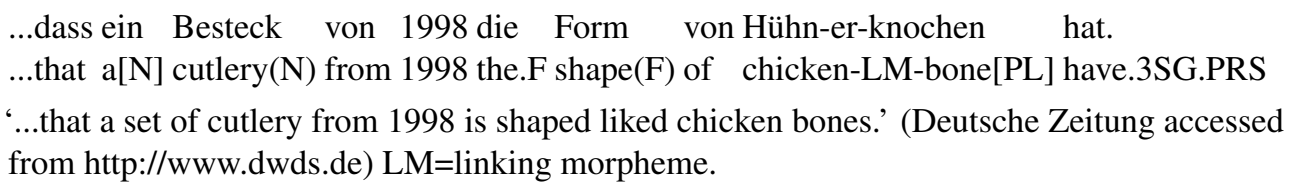

However, counting of individual cutlery items is blocked and felicity is considerably reduced when directly attaching numerical expressions to mass collective artifacts.

2 From a petroleum technology journal, (Adekomaya 2013).

3 Hensperger, B. and J. Kaufmann. The Ultimate Rice Cooker Cookbook, Harvard Common Press, 2003. p. 23. 
The resistance of collective artifacts to these kinds of coercion is puzzling. As the alternative description object mass noun for the English nouns of this type suggests, there are clearly identifiable 'atomic' entities in the denotations of such nouns (e.g., single items of furniture, footwear or jewelry), so it may be perplexing why these cannot be accessed via coercion. Furthermore, mass collective artifacts tend to describe superordinate categories. For example, furniture, as a label for a superordinate category, comprises basic level categories labeled by count nouns like table, chair, etc., and we also have a level of different kinds of furniture like bedroom furniture, a superordinate kind. Therefore, it is also puzzling why these levels cannot be accessed for grammatical counting in coerced environments. Notice, for example, that the superordinate, or more neutrally, collective, nature of the denotations of nouns like furniture cannot explain the resistance to the coercive operations in question. There are superordinate/collective artifact count nouns like the Finnish examples (3b), (4b), (5b). Furthermore, even English has examples like these such as vehicle that denote superordinate categories, which comprise different subordinate level categories like car, truck, bus, motorcycle, and which are straightforwardly acceptable in numerical count constructions, in which either specific ordinary individuals (of the same (sub)kind) or different (sub)kinds are counted, as shown in (13):

(13) The brief for the government-backed project is to produce four vehicles ranging in size from the Ford Fiesta to the Vauxhall Cavalier. ${ }^{4}$

In this paper, we will focus on restrictions to mass-to-count packaging coercion and will leave aside a treatment of mass-to-kind coercion. (However, for a related treatment for this latter type of shift, see Sutton \& Filip 2016b.)

\section{Background}

\subsection{Domains, semantic types, and extensive measure functions}

We assume a familiarity, in the following, with the classical mereological approach to nominal semantics as first suggested in Link 1983, in which noun denotations are represented as Boolean semi-lattices minus the bottom element, closed under sum. Link's (1983) account posited two separate domains for count and mass denotations. However, following Krifka (1989), most now reject Link's (1983) dual domain assumption. Krifka (1989) adopted a single domain, and suggested that the meanings of all (concrete) count nouns are of a different semantic type to mass

4 http://www.independent.co.uk/arts-entertainment/motoringfamily-planning-1577828.html (accessed: 15.03.2016). 
Counting in context

nouns. For example, lexical entries are given for cow in (14) and mud in (15):

$$
\begin{aligned}
\llbracket \operatorname{cow} \rrbracket & =\lambda n \lambda x[\mathbf{C O W}(x) \wedge \mathbf{N U}(\mathbf{C O W})(x)=n]_{\langle n,\langle e, t\rangle\rangle} \\
\llbracket \operatorname{mud} \rrbracket & =\lambda x[\mathbf{M U D}(x)]_{\langle e, t\rangle}
\end{aligned}
$$

In (14), NU is 'natural unit' extensive measure function that ensures that singular count nouns are represented by quantized predicates (predicates for which no two entities in their denotations are proper parts of one another). ${ }^{5}$

\subsection{Counting-context sensitivity: Rothstein 2010}

Rothstein (2010) follows Krifka (1989) in adopting a type-based distinction, however Rothstein's (2010) major contribution is to address the data that were problematic for Krifka's (1989) account. As pointed out in Zucchi \& White 1996, 2001 (and p.c. Barbara Partee), there are count nouns that do not come in natural units e.g., line, fence, twig, and that are divisive (down to some minimal level). As such, predicates representing these nouns are not quantized, and so should not be apt for counting.

To counter this problem, Rothstein (2010) introduces the concept of a counting context. Count nouns denote a set of entity and counting context pairs. The entities in these pairs count as 'one' at that context and form a disjoint set (at least in default cases). In (16-18) Rothstein's formalism is converted into one which makes the comparison with Krifka's more transparent. The variable $x$ is of type $\langle e\rangle$. Variables in bold, $\mathbf{x}$, are of type $\langle e \times k\rangle$, where $k$ is the type of counting contexts. $\pi_{1}$ and $\pi_{2}$ are such that $\pi_{1}\left(\left\langle a, k_{1}\right\rangle\right)=a$ and $\pi_{2}\left(\left\langle a, k_{1}\right\rangle\right)=k_{1}$. Counting contexts, like rootpredicates can be thought of as type $\langle e, t\rangle$ (i.e. subsets of the domain of entities). Whereas Krifka uses the extensive measure function NU, Rothstein's innovation is to essentially make this function context-sensitive. $\pi_{2}(\mathbf{x})$ (the counting context) applies to $\pi_{1}(\mathbf{x})$ (an entity), and so selects for potentially different entities for counting depending on the context.

$$
\begin{aligned}
\llbracket \operatorname{cow} \rrbracket & =\lambda \mathbf{x}\left[\operatorname{COW}\left(\pi_{1}(\mathbf{x})\right) \wedge\left(\pi_{2}(\mathbf{x})\right)\left(\pi_{1}(\mathbf{x})\right)\right]_{\langle e \times k, t\rangle} \\
\llbracket \text { fence } \rrbracket & =\lambda \mathbf{x}\left[\operatorname{FENCE}\left(\pi_{1}(\mathbf{x})\right) \wedge\left(\pi_{2}(\mathbf{x})\right)\left(\pi_{1}(\mathbf{x})\right)\right]_{\langle e \times k, t\rangle} \\
\llbracket \operatorname{mud} \rrbracket & =\lambda x[\operatorname{MUD}(x)]_{\langle e, t\rangle}
\end{aligned}
$$

Countable entities are indexed to counting contexts. For example, in (17), at a counting context $k_{1}$, entities in entity-context pairs must be in the denotation of FENCE, and must be in the denotation of the counting context $k_{1}$. The result of this is that, at different contexts, there may be different answers to the question,

$5 \forall P[\mathbf{Q U A}(P) \leftrightarrow \forall x \forall y[P(x) \wedge P(y) \rightarrow \neg x \sqsubset y]]$ (Krifka 1989: 78) 
How many fences are there? For example, if FENCE $=\{a, b, c, d, \ldots, a \sqcup b \sqcup c \sqcup d\}$, $k_{1}=\{a, b, c, d\}$ and $k_{2}=\{a \sqcup b \sqcup c \sqcup d\}$, then:

$$
\begin{aligned}
& \llbracket \text { fence } \rrbracket^{k_{1}}=\left\{\left\langle a, k_{1}\right\rangle,\left\langle b, k_{1}\right\rangle,\left\langle c, k_{1}\right\rangle,\left\langle d, k_{1}\right\rangle\right\} \\
& \llbracket \text { fence } \rrbracket^{k_{2}}=\left\{\left\langle a \sqcup b \sqcup c \sqcup d, k_{2}\right\rangle\right\}
\end{aligned}
$$

The result is that there would be four fences at $k_{1}$ and one fence at $k_{2}$.

\subsection{Overlap makes counting go wrong: Landman 2011}

A key concept for Landman (2011) is a generator set, namely, a set of entities that generates a noun's denotation via upward closure under sum. Count nouns have non-overlapping generator sets. For example, the (chosen) generator set for chair will be the disjoint set of the single individual chairs. All mass nouns have overlapping generator sets. Overlap in the chosen generator set (that is not ignored) makes counting go wrong. Counting goes wrong, because of all the ways to resolve overlap, there is no single answer to the question of 'How many $\mathrm{X}$ are there?'.

However, as Landman (2011) argues, two kinds of mass nouns can be distinguished in terms of properties of their generator sets. Neat mass nouns include the nouns we have called 'mass collective artifacts' (furniture, kitchenware). Mess mass nouns are prototypical mass nouns ( $m u d$, blood). Neat mass nouns have non-overlapping minimal generator sets. For example, in the case of kitchenware, minimal generators include single cups and single saucers. Generators also include sums (e.g., a sum of a cup and a saucer). Mess mass nouns, on the other hand, have overlapping minimal generators (and so a forteriori overlapping generators).

Formally, how counting succeeds or goes wrong is defined in terms of variants of generator sets. Variants are maximally disjoint subsets of generator sets. Counting goes wrong if the cardinality of variants of a generator set differs depending on the variant. Since there can be only one maximally disjoint subset of a disjoint set, namely the set itself, nouns with disjoint generator sets are count. For mass nouns, the situation is different. For example, suppose that the generator set for kitchenware is such that: $\operatorname{gen}\left(\mathrm{K}_{-}\right.$WARE $)=\{$cup, saucer, cup $\sqcup$ saucer $\}$, then there are two possible variants $V_{1}$ and $V_{2}$, as below. These give inconsistant counting results, hence counting goes wrong.

$$
\begin{array}{r}
\mid V_{1}\left(\operatorname{gen}\left(\mathrm{K}_{-} \text {WARE }\right)\right)|=|\{\text { cup, saucer }\} \mid=2 \\
\left|V_{2}\left(\operatorname{gen}\left(\mathrm{K}_{\text {_WARE }}\right)\right)\right|=\mid\{\text { cup } \sqcup \text { saucer }\} \mid
\end{array}
$$


Counting in context

\section{Analysis: Rothstein-Landman synthesis}

\subsection{Challenges for Rothstein's and Landman's accounts}

The main focus of Rothstein's (2010) account is why some nouns, that are divisible and cumulative (e.g. fence), exhibit count grammatical properties. This leaves the analysis of mass nouns open for further development within a broadly Rothsteinian framework. In essence, the position in Rothstein 2010 is that count nouns are count nouns because they are indexed to counting contexts (have "semantic atoms"). Mass nouns are mass nouns because they are not of this semantic type. Although an account based principally on a difference of semantic type is not objectionable when giving an explanation of the data at one level of detail, taking such an approach potentially masks a deeper explanation for explaining cross- and intralinguistic patterns in count/mass variation, and restrictions on mass-to-count coercion. For example, if furniture is, for all intents and purposes, like mud, being of type $\langle e, t\rangle$, then it is puzzling why we find so many instances of furniture-denoting count nouns cross-linguistically, but relatively few for mud/blood/air-denoting nouns. ${ }^{6}$ However, since Rothstein (2010: 394) explicitly leaves the issue of crosslinguistic count/mass variation aside for future research, this paper could be viewed as a proposal to enrich a framework based on Rothstein-style counting contexts in order to get a better grip on these data.

A further unresolved puzzle on Rothstein's (2010) account, one that she is aware of (Rothstein 2015), is why object mass nouns (our mass collective artifacts) resist mass-to-count coercion. Indeed, this is also a puzzle, given Landman's (2011) framework. It is worth noting, moreover, that the natural atomicity of such nouns actually exacerbates this puzzle. After all, if any mass nouns should be easily coerced into a count reading, one would expect naturally atomic mass nouns to be good candidates, since the obvious units for counting are already there in the denotation.

Landman (2011) observes that Dutch lexicalizes both a count and a mass furniture-denoting noun, respectively meubel(s) and meubilair. Unlike on Rothstein's (2010) approach, the difference, semantically, between meubel and meubilair, for Landman, is not a matter of a difference in semantic type, but in the disjointness/overlap of their generator sets. What is missing, in Landman 2011, however, is a detailed account of why we find so much count/mass variation in the class of collective artifact nouns, but very little in prototypical count nouns (e.g. cat) and prototypical mass nouns (e.g. $\mathrm{mud}$ ).

6 On the other hand, Rothstein argues that furniture-like nouns are naturally atomic, unlike mud-like nouns. In principle, then, one could surmise that naturally atomic nouns display crosslinguistic count/mass variation. However, this is not a necessary or sufficient criterion for predicting count/mass variation, since there are stably count naturally atomic nouns (cat, chair), and mass-counterparts of non-naturally atomic nouns (e.g. fencing for fence, and hedging for hedge). 


\subsection{A synthesis of Landman's variants and Rothstein's counting contexts}

For both Rothstein (2010) and Landman (2011) the appeal to context is of key importance. Whereas for Rothstein it is a central element of her theoretical apparatus, for Landman it is prominent at a pretheoretical level. ${ }^{7}$ This is clear in his treatment of neat mass nouns, which relies on considerations like the following one: "the teapot, the cup, the saucer, the cup and saucer all count as kitchenware and can all count as one simultaneously in the same context ... The generating set of furniture overlaps, but the overlap is only vertical: a sum and its parts count as one simultaneously." (Landman 2011: 34-35). There is, however, a formal mechanism to remove overlap on Landman's account, namely a variant. Recall that a variant is a maximally disjoint subset of the generator set, which represents one possible schema for counting.

Now compare this with fence-like nouns on Rothstein's (2010) account. There, recall, the 'root' denotation of the predicate FENCE was also overlapping. Counting fences is made possible, because counting contexts intersect with the denotation of FENCE, yielding a disjoint set of fences, or, in Rothstein's terms, a set of entitycontext pairs, the entities in which, in default cases, form a disjoint set.

Despite the differences in the motivation and formal characterisations of variants and counting-contexts, their result is the same, namely, to map a non-disjoint set onto a disjoint one. The formal encoding of this similarity is easily achieved. We introduce the NULL COUNTING CONTEXT to model 'context' in Landman's "can all count as one simultaneously in the same context". Counting contexts and null counting contexts can now be inter-defined. To simplify matters, we assume that counting contexts are indices on interpretations of predicates. We assume a domain of counting contexts $\mathscr{C}=\left\{c_{0}, c_{1}, \ldots, c_{n}\right\}$ such that $c_{0}$ is the null counting context, and $c_{1}, \ldots, c_{n}$ are default counting contexts, roughly Landman's variants.

$$
X_{c_{0}}=\bigcup X_{c_{i}} \text { computed from all } c_{i>0} \in \mathscr{C}
$$

In words, the interpretation of a predicate at the null context is the union of the interpretations of the predicate at all counting contexts (i.e. variants). An example of this is depicted in Figure 1. Suppose that the denotation of entities that could count as 'one' for kitchenware is \{pestle, mortar, pan, lid, pan $\sqcup$ lid, pestle $\sqcup$ mortar\}. At any given counting context, we get a maximally disjoint subset of this set. For instance, in $c_{1}$, it could be \{pestle, mortar, pan $\sqcup$ lid $\}$. This, intuitively could represent, for example, the denotation of the German count noun Küchengerät ('Kitchenware', lit. 'kitchen.device') in $c_{1}$. At the null counting context, $c_{0}$, however, the set is not disjoint, because what can count as 'one' overlaps. For the same reasons as outlined in Landman 2011, counting goes wrong. This overlapping set intuitively forms, for

7 For a more detailed discussion of this point see Sutton \& Filip 2016a. 


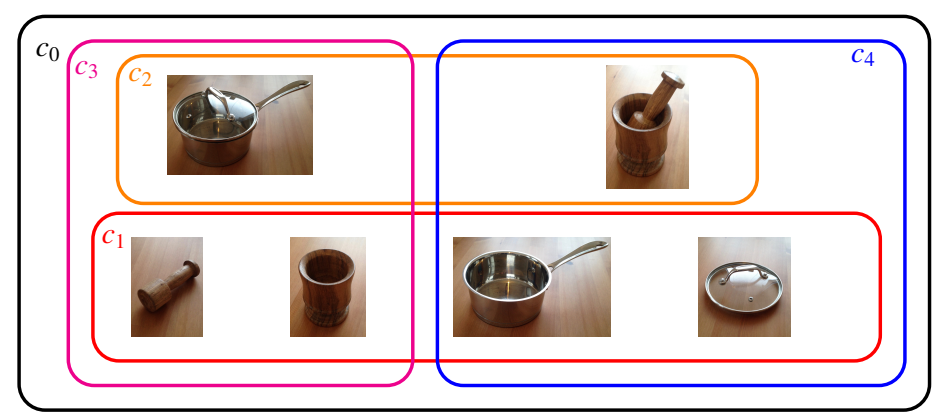

Figure 1 Kitchenware: Denotations for specific counting contexts and the null counting context

example, the counting base for the English mass noun kitchenware. (The upward closure under sum of this set would be the full denotation for kitchenware.)

In summary, the notion of counting context in Rothstein 2010 roughly corresponds to the notion of a variant in Landman 2011. Also Landman's contexts, in which what counts as 'one' overlaps simultaneously, can be modelled in terms of all the counting contexts, which remove overlap. The immediate advantage of combining these two accounts is that we can now potentially give a semantics that covers not only prototypical count (boy) and mass (water) nouns, but also fence-like count nouns and furniture-like mass nouns at a level of detail that has not yet been provided in extant accounts. In Section 5, we will add more details to this basic framework and will thereby derive the correct predictions for mass/count variation for collective artifacts.

\section{Proposal: Counting in context}

We follow Krifka (1989) in assuming that lexical entries for concrete nouns contain both qualitative and quantitative criteria. Krifka's quantitative element was his natural unit function. We assume a function IND that maps number neutral predicates (of type $\langle e, t\rangle$ ) to the entities that count as 'one' for that predicate. What counts as 'one' is context-sensitive, which is rendered by having IND introduce a counting context argument, so IND will be of type $\langle\langle e, t\rangle,\langle c,\langle e, t\rangle\rangle\rangle$. At the null counting context, IND sets include everything that could count as 'one' for that predicate.

To make clear what we mean by counting as 'one', some toy examples are given in Table 1 which show the number neutral predicate denotation $P$, an example of $\operatorname{IND}(P)$ at some specific counting contexts, and at the null counting context. As Table 1 shows, the effect of IND and counting contexts on number neutral predicates is not identical on different classes of noun denotations. This is because IND is 


\begin{tabular}{ll} 
CAT & $=\{$ felix, tibbles, garfield, .., felix $\sqcup$ tibbles $\sqcup$ garfield $\}$ \\
IND $(\text { CAT })_{c_{i} \geq 0}$ & $=\{$ felix, tibbles, garfield $\}$ \\
\hline K_WARE & $=\{$ pestle, mortar, pan, lid,$\ldots$, pestle $\sqcup$ mortar $\sqcup$ pan $\sqcup$ lid $\}$ \\
IND $(\text { K_WARE })_{c_{3}}$ & $=\{$ pan $\sqcup$ lid, pestle, mortar $\}$ \\
IND $(\text { K_WARE })_{c_{4}}$ & $=\{$ pan, lid, pestle $\sqcup$ mortar $\}$ \\
IND $(\text { K_WARE })_{c_{0}}$ & $=\{$ pestle, mortar,pan, lid, pestle $\sqcup$ mortar, pan $\sqcup$ lid $\}$ \\
\hline FENCE & $=\left\{f_{1}, f_{2}, f_{3} \ldots, f_{1} \sqcup f_{2} \sqcup f_{3}\right\}$ \\
IND $(\text { FENCE })_{c_{1}}$ & $=\left\{f_{1}, f_{2}, f_{3}\right\}$ \\
IND $(\text { FENCE })_{c_{2}}$ & $=\left\{f_{1} \sqcup f_{2}, f_{3}\right\}$ \\
IND $(\text { FENCE })_{c_{0}}$ & $=\left\{f_{1}, f_{2}, f_{3}, f_{1} \sqcup f_{2}, \ldots\right\}$ \\
\hline $\operatorname{MUD}$ & $=\left\{\ldots, m_{1} \sqcup m_{2} \sqcup m_{3}\right\}$ a vague set \\
IND $(\text { MUD })_{c_{i \geq 0}}$ & $=\varnothing$
\end{tabular}

Table 1 Examples of number-neutral denotations, $P$ and denotations of $\operatorname{IND}(P)$

assumed to be sensitive to the perceptual and functional properties of predicates and their denotations. When a predicate denotes entities that are conceived of as being functionally and/or perceptually individuable, i.e. occurring in salient non-overlapping entities that count as one (e.g. CAT), the IND set is a disjoint set at all counting contexts and the null counting context (e.g. the set of single cats). For kitchenware- and fence-like predicates, the entities that count as 'one' vary from context to context. At some contexts sums count as 'one'. At other contexts, proper parts of sums each count as 'one'. Hence, the IND set evaluated at the null counting context is not disjoint. For substance denoting nouns such as $m u d$, we claim that there is, intuitively, nothing that clearly counts as 'one', even at some specific context (barring highly specialized scientific or technical contexts and uses that differ from our ordinary everyday use). This is why we give an empty denotation for IND(MUD) at all counting contexts (so also at the null counting context). Alternatively, this could be undefined, rather than empty. In this last respect, we depart from Landman 2011 in which the generator sets for substances have overlapping minimal generators. We make no commitments to the nature of the minimal parts of mud-like nouns in our semantics, even relative to some context.

Now we have all the basic ingredients we need to give the lexical entries for count and mass nouns. We follow Landman 2011, 2016 in giving lexical entries as ordered pairs and we distinguish these in terms of the terminology body and (counting) base. For us, the BODY is the NUMBER NEUTRAL noun predicate. The semantic properties of the counting base determine whether a noun is grammatically 
countable. Unlike Landman 2011, 2016, we do not require counting bases to generate bodies (a property central to Landman's theory).

Importantly, we distinguish, semantically, between predicates for substances and objects. One of the reasons is that pre-linguistic infants can distinguish substances from objects (Soja, Carey \& Spelke 1991). While this does not necessarily mirror the mass/count distinction, because mass nouns like furniture have discrete objects in their denotation, rather than undifferentiated stuff, it does mirror the distinction between what we can and cannot intuitively individuate on perceptual and functional grounds. For example, with basic lexical nouns such as cat, chair, kitchenware, fence, fencing, we can, relative to a context, determine, either on the basis of perceptual properties of their referents, or on the basis of functional criteria, what counts as 'one' ('item'/ 'piece' in the case of nouns like kitchenware and fencing). In contrast, the denotations of basic lexical nouns such as mud and blood cannot be so individuated. (Nota bene, some native speakers conceptualize fencing as substance denoting, just like mud, blood, however.) This conceptual difference is prelinguistic, and we propose to encode this distinction in the lexical entries of concrete nouns as shown in (24). In words, the counting bases for substance denoting nouns are the same as the bodies, i.e., number neutral predicates. The counting bases of all other nouns will be the IND function applied to the number neutral predicate.

$$
\llbracket \mathrm{n} \rrbracket^{c_{i}}= \begin{cases}\langle N, N\rangle & \text { if } \operatorname{IND}(N)=\varnothing \text { at all counting contexts } c_{i} \\ \langle N, \operatorname{IND}(N)\rangle & \text { otherwise }\end{cases}
$$

Following Krifka (1989) and Rothstein (2010), on our account, there is a typal distinction between count and mass nouns. However, the type difference will not itself draw the count/mass boundary. Instead, properties of counting bases will. Inspired by Landman's (2011) overlap simultaneously in the same context, on our account, mass nouns are saturated with the null context $c_{0}([+\mathrm{C}]$ indicates COUNT):

$$
\llbracket \mathrm{n} \rrbracket^{c_{i}}= \begin{cases}\left\langle N, \mathbf{c} \_ \text {base }(N)_{c_{i}}\right\rangle & \text { if } \mathrm{n} \text { is }[+\mathrm{C}] \\ \left\langle N, \mathbf{c} \_ \text {base }(N)_{c_{0}}\right\rangle & \text { if } \mathrm{n} \text { is }[-\mathrm{C}]\end{cases}
$$

Notice, however, that the typal distinction is eliminated whenever an $\mathrm{N}$ is used in context. Mass nouns come pre-saturated with the null context. As such, their context argument comes pre-filled, and so they reduce to being of type $\langle e, t\rangle$ whereas count nouns, which do not come pre-saturated, are of type $\langle c,\langle e, t\rangle\rangle$. However, as soon as a count noun is used, the context of utterance saturates the context argument and so count nouns used in context, like mass nouns, will be of type $\langle e, t\rangle$.

Following Landman 2011, 2016, we view disjointness/non-disjointness as a major semantic property that underpins the count/mass distinction:

If c_base $(N)$ is disjoint, then counting is possible. If c_base $(N)$ is not disjoint, then counting goes wrong. 
This means that, for some predicates, it could be the case that their countability status changes depending on the context at which the counting base is fixed. If a predicate has an overlapping counting base at the null context, but a disjoint counting base at all other counting contexts, then we expect a mass noun in the former case, and a count noun in the latter. This use of variation between null and other counting contexts is the device that predicts count/mass variation. We now give four examples.

\subsection{Prototypical count nouns (e.g. cat in English)}

As a count noun, cat will be indexed to the counting context of evaluation. This is shown in (28). The counting base in (28) is the set of individual cats, which is a disjoint set, and hence counting is possible.

$$
[[\mathrm{cat}]]^{c_{i}}=\lambda x \cdot\left\langle\mathrm{CAT}(x), \mathbf{I N D}(\mathrm{CAT})\left(c_{i}\right)(x)\right\rangle
$$

However, suppose we were to try to treat cat as a mass noun in the lexicon. This would mean evaluating the base at the null counting context as in (29).

$$
[[\mathrm{cat}]]^{c_{i}}=\lambda x \cdot\left\langle\operatorname{CAT}(x), \mathbf{I N D}(\mathrm{CAT})\left(c_{0}\right)(x)\right\rangle
$$

Crucially, the result is still that counting is possible. This is because, if the counting base, the disjoint set of individual cats, is the same disjoint set across all counting contexts, then the counting base at the null context will be disjoint too. This predicts that cat-like nouns will be stably count crosslinguistically.

\subsection{Collective artifacts (e.g. kitchenware in English)}

As a mass noun, the English noun kitchenware is indexed to the null counting context. This is shown in (30). The counting base in (30) will be the set of all things that could count as one item of kitchenware. As shown in Table 1, this is not disjoint, so counting is not possible.

$$
[[\text { kitchenware }]]^{c_{i}}=\lambda x .\left\langle\mathrm{K}_{-} \mathrm{WARE}(x), \mathbf{I N D}\left(\mathrm{K}_{-} \mathrm{WARE}\right)\left(c_{0}\right)(x)\right\rangle
$$

However, suppose we were to try to give KITCHENWARE a count noun entry in the lexicon. This would mean evaluating the base at the counting context of utterance. And, most importantly, this results in a disjoint counting base, and so something that can be counted. In other words, we would have a lexical entry for a noun such as the German count noun Küchengerät ('kitchenware', lit. 'kitchen.device'). This is shown in (31).

$$
[[\text { Küchengerät }]]^{c_{i}}=\lambda x \cdot\left\langle\mathrm{K}_{-} \operatorname{WARE}(x), \mathbf{I N D}\left(\mathrm{K}_{-} \mathrm{WARE}\right)\left(c_{i}\right)(x)\right\rangle
$$


The same applies to furniture versus the Finnish count noun huonekalu ('furniture') as in (3a) and (3b). A switch between the possibility and impossibility of grammatical counting for words that share the same underlying concept depends on whether we evaluate their counting base at the null counting context (provided in their lexical entries) or counting context of utterance. In this way, we account for the cross- and intralinguistic variation in the lexical encoding of collective artifacts: There is, for such nouns, some lexical choice. Saturating with the null counting context yields something conceptually mass. Evaluating at the counting context of utterance yields something conceptually count.

\subsection{Non-prototypical count nouns (e.g. fence in English)}

As a count noun, fence will be indexed to the counting context of utterance. This is shown in (32). The base in (32) will be the set of all entities that count as one fence in that context. As shown in Table 1, this is disjoint, so counting is possible.

$$
[[\text { fence }]]^{c_{i}}=\lambda x \cdot\left\langle\operatorname{FENCE}(x), \mathbf{I N D}(\operatorname{FENCE})\left(c_{i}\right)(x)\right\rangle
$$

However, suppose we were to try to treat FENCE as a mass noun in the lexicon by evaluating the counting base at the null counting context. This results in a nondisjoint base, and so counting is not possible, since across contexts, what counts as two fences in one context may count as one fence in another and the null counting context includes a union of both the sum and its parts. In other words, we would have a lexical entry for a mass noun such as fencing. This is shown in (33).

$$
[[\text { fencing }]]^{c_{i}}=\lambda x \cdot\left\langle\operatorname{FENCE}(x), \mathbf{I N D}(\operatorname{FENCE})\left(c_{0}\right)(x)\right\rangle
$$

The fact that we see a switch between the possibility and impossibility of counting depending on whether we evaluate at the null counting context or not predicts count/mass variation intra- and crosslinguistically. As with KITCHENWARE denoting nouns, there is, for such nouns, a degree of lexical choice. Saturating with the null counting context yields something conceptually mass. Evaluating at the counting context of utterance yields something conceptually count.

\subsection{Prototypical mass nouns (e.g. mud in English)}

Substance nouns like mud get a different treatment. As shown in (34) the base is the same as the body, namely, the denotation of the number neutral predicate MUD. This is not disjoint, so counting is not possible.

$$
[[\mathrm{mud}]]^{c_{i}}=\lambda x \cdot\langle\operatorname{MUD}(x), \operatorname{MUD}(x)\rangle
$$

However, notice that the base is not indexed to a counting context null or otherwise. This predicts that mud-like nouns will be stably mass intra- and crosslinguistically. 


\subsection{Summary}

The exciting consequence of this framework for the count/mass distinction is that the semantic properties of counting bases, in terms of overlap and non-overlap, can be used to predict when we should expect to find intra- and crosslinguistic count/mass variation. We have argued that nouns fall into at least three broad classes. Prototypical count nouns (e.g. cat, chair, as in English) have disjoint counting bases and are stably count. Kitchenware- or furniture-like nouns (collective artifacts) and fence-like nouns have overlapping counting bases. Languages can either encode the resolution of this overlap in context, which leads to a lexicalization as a count noun fence, or leave it unresolved, which results in a lexicalization as a mass noun furniture. This optionality, we claim, explains the prevalence of count/mass variation within these noun classes. Substances (e.g. mud, blood, air) are distinct from all other concrete nouns on the basis of our prelinguistic distinction between objects and substances. Nothing intuitively counts as one for these nouns, and so they are stably mass.

\section{Proposal: Coercion in context}

In this section, we will provide a highly simplified semantics for two types of classifier constructions: CONTAINER-readings ${ }^{8}$ of expressions such as bowl of which, when applied to e.g. apples would mean BOWL CONTAINING APPLES); UNIT-EXTRACTING classifiers such as item of. We will then locate one difference in how implicitly supplied classifiers in cases of coercion differ from explicitly (i.e. lexically) provided ones.

\subsection{Container and unit-extracting classifiers}

Like other nouns, the lexical entries for container classifiers (e.g. glass of, bottle of) will have a body (corresponding to the number neutral noun predicate denotation) and a counting base. ${ }^{9}$ When combined with their argument nouns, they supply their own bodies, so, for example, the body of the classifier-noun phrase bottle of water will be the number neutral property, BOTTLE. The counting bases of classifiers combine with contents from the counting base of the argument noun. For example, bottles containing water. Container classifiers are also sensitive to plurality and the mass/count distinction, they require cumulative predicates (mass or bare plural) as

8 We leave other classifier readings, such as contents and portion readings for future research.

9 Much inspiration here is from Landman 2016. Our proposal differs from his, however. For Landman, the condition that the base generates the body under sum is an essential part of the theory. 
Counting in context

their input. We represent this in (35):

(35)

$C L_{\text {container }}(P)= \begin{cases}\left\langle\operatorname{body}(C L), \mathbf{c} \_ \text {base }(C L)\left(\mathbf{c} \_ \text {base }(P)\right)\right\rangle & \text { if } P \text { is }[+P L] \text { or }[-C] \\ \perp & \text { if } P \text { is }[+S G,+C]\end{cases}$

For example, a derivation for glasses of wine is given in (36). ${ }^{10}$

$$
\begin{aligned}
& \llbracket \text { glasses of } \rrbracket^{c_{i}}= \\
& \lambda P . \lambda x \cdot \exists y \cdot\left\langle(\mathrm{GL})(x),{ }^{*} \operatorname{IND}(\mathrm{GL})\left(c_{i}\right)(x) \wedge \operatorname{CONTS}(x)=y \wedge \mathbf{c} \_ \text {base }(P)(y)\right\rangle \\
& \llbracket \text { glasses of wine } \rrbracket^{c_{i}}= \\
& \lambda x . \exists y \cdot\left\langle(\mathrm{GL})(x),{ }^{*} \operatorname{IND}(\mathrm{GL})\left(c_{i}\right)(x) \wedge \operatorname{CONTS}(x)=y \wedge \operatorname{WINE}(y)\right\rangle
\end{aligned}
$$

Entries for bowls of, apples, and bowls of apples are given in (37).

$$
\begin{aligned}
& \llbracket \text { bowls of } \rrbracket^{c_{i}}= \\
& \lambda P . \lambda x \cdot \exists y \cdot\left\langle(\operatorname{BOWL})(x),{ }^{*} \operatorname{IND}(\operatorname{BOWL})\left(c_{i}\right)(x) \wedge \operatorname{CONTS}(x)=y \wedge \mathbf{c} \_ \text {base }(P)(y)\right\rangle \\
& \llbracket \text { apples } \rrbracket]^{c_{i}}=\lambda z \cdot\left\langle\operatorname{APPLE}(z),{ }^{*} \operatorname{IND}(\operatorname{APPLE})\left(c_{i}\right)(z)\right\rangle \\
& \llbracket \text { bowls of apples } \rrbracket^{c_{i}}= \\
& \lambda x \cdot \exists y \cdot\left\langle(\operatorname{BOWL})(x),{ }^{*} \operatorname{IND}(\operatorname{BOWL})\left(c_{i}\right)(x) \wedge \operatorname{CONTS}(x)=y \wedge{ }^{*} \operatorname{IND}(\operatorname{APPLE})(y)\right\rangle
\end{aligned}
$$

Classifiers apply to cumulative predicates, either mass or bare plural, and yield a complex count predicate. This is because the counting base of the classifier noun (BOTTLE, BOWL) is a disjoint set, and we count in terms of units specified by it (BOTTLES CONTAINING WINE, BOWLS CONTAINING APPLES).

Unit-extracting classifiers (item of, piece of) are different from container classifiers. First, they select only mass nouns. For example, a piece of chair is only felicitous (if at all), if the argument noun chair, which is inherently count, is first coerced into a mass interpretation. ${ }^{11}$ Second, they are underspecified with respect to their unit for counting, and so adopt the individuation schema from the counting base of the argument noun (provided the two are compatible). Third, explicitly provided unit-extracting classifiers also need a mechanism for extracting units from mass noun denotations. Within our formalism, this is easily represented as a counting-context substitution rule, $\left[c_{0} / \varnothing \mapsto c_{i}\right]$. For nouns such as the English furniture, the rule replaces the null counting context, $c_{0}$, with the counting context of utterance $c_{i}$. For nouns such as the English mud, which have no counting context in the base, the rule adds in the counting context of utterance $c_{i}$. Explicitly provided unit-extractors supply their own counting contexts: they forcibly insert the counting context of utterance into the interpretation of the whole unit-extracting phrase. The above

$10 \mathrm{GL}$ is the predicate for $\operatorname{GLASS} \operatorname{CONTS}(x)=y$ means that the contexts of $x$ is $y$.

11 We focus here on pseudo-partitive (measure) constructions like a piece of chair, and set aside partitive constructions like a piece of the chair, which are straightforwardly acceptable, of course. 
assumptions are represented in (38) and a sample derivation is given in (39).

$$
\begin{aligned}
& \mathrm{UE}(P)_{c_{i}}= \begin{cases}\left\langle\operatorname{body}(P), \operatorname{base}(P)_{\left[c_{0} / \varnothing \mapsto c_{i}\right]}\right\rangle & \text { if } P \text { is }[-C] \\
\perp & \text { otherwise }\end{cases} \\
& \llbracket \text { item/piece of } \rrbracket^{c_{i}} \quad=\lambda P . \lambda x \cdot\left\langle\operatorname{body}(P)(x), \operatorname{base}\left(P_{\left[c_{0} / \varnothing \mapsto c_{i}\right]}(x)\right)\right\rangle \\
& \llbracket \text { item/piece of furniture } \rrbracket^{c_{i}}=\lambda x \text {. }\left\langle\mathrm{FURN}(x), \operatorname{IND}(\mathrm{FURN})\left(c_{0}\right)_{\left[c_{0} \mapsto c_{i}\right]}(x)\right\rangle \\
& =\lambda x \cdot\left\langle\operatorname{FURN}(x), \operatorname{IND}(\operatorname{FURN})\left(c_{i}\right)(x)\right\rangle
\end{aligned}
$$

The resulting complex noun in (39) is also grammatically countable, since the null counting context from furniture has been replaced by the counting context of utterance as part of the semantics of the expression item of. From context to context, it may vary what exactly counts as one item of furniture, but at any context, this overlap will be resolved.

Unit extractors can also work for substance nouns, as shown in (40). The difference is that there is no IND provided by the base for mud. Intuitively, this captures the idea that there seems to be a great deal more variation in what counts as a piece of mud compared with what counts as a piece of furniture.

$$
\begin{aligned}
\llbracket \text { piece of mud } \rrbracket^{c_{i}} & \left.=\lambda x \cdot\left\langle\operatorname{MUD}(x), \operatorname{MUD}_{\left[\varnothing \mapsto c_{i}\right]}(x)\right)\right\rangle \\
& =\lambda x \cdot\left\langle\operatorname{MUD}(x), \operatorname{MUD}\left(c_{i}\right)(x)\right\rangle
\end{aligned}
$$

\subsection{Mass-to-count coercion: Implicit classifier concepts}

As we saw when presenting the data in Section 2, the implicit addition of classifier concepts in cases of mass-to-count coercion cannot be identical to providing an explicit unit extractor. For example, three furnitures cannot be coerced to mean 'three items of furniture'. Our account allows a simple explanation of this fact. We have argued that explicit unit-extractors forcibly insert the counting context of utterance into the interpretation of the whole unit-extracting phrase. This involved a context-substitution procedure. However, in cases of coercion, we use the context provided in the lexical entry of the argument noun, because there is no explicit classifier to switch contexts. In other words, the implicit unit-extractor cannot perform the 'heavy handed' semantic operation of overwriting the null context with the context of utterance. This is shown in (41) and the effect on furniture is shown in (42).

$$
\begin{gathered}
P_{\mathrm{UE} \text { coerced }}= \begin{cases}\langle\operatorname{body}(P), \operatorname{base}(P)\rangle & \text { if } P \text { is }[-C] \\
\perp & \text { otherwise }\end{cases} \\
\llbracket \text { furniture }_{\mathrm{UE} \text { coerced }} \rrbracket^{c_{i}}=\lambda x .\left\langle\operatorname{FURN}(x), \operatorname{IND}(\mathrm{FURN})\left(c_{0}\right)(x)\right\rangle
\end{gathered}
$$

Unlike in (39), in (42) the null context in the base is not replaced with the counting context of utterance. Hence, the base is not disjoint, and so counting is not possible. 
Explicitly provided container classifiers such as glass (of) do not include a context rewrite rule (see (36)), and so an implicit addition of a CL concept will result in a similar result as in the explicit case. The one difference, we suggest, is that any plural morphology in the coerced noun gets interpreted as plurality in the base. This is shown in (43).

$$
{ }^{(*)} P_{\mathrm{CL} \text { coerced }}= \begin{cases}\left\langle\boldsymbol{\operatorname { b o d }}(C L),{ }^{(*)} \mathbf{b a s e}(C L)(\operatorname{base}(P))\right\rangle & \text { if } P \text { is }[-C] \\ \perp & \text { otherwise }\end{cases}
$$

For example, wines, coerced with GL (glass), with the entry in (43), would yield the same result as (36). Equally, for an appropriate entry for a coerced insertion of SAMPLE, we could derive three muds as 'three SAMPLES OF mud' as in (10).

\subsection{Summary}

In this section we have given a framework that accommodates data that, to our knowledge, are intractable for other theories. Namely, we can explain why collective aggregates resist unit-extracting mass-to-count coercion. We have also shown why it is possible to coerce mass nouns such as mud into coerced readings such as 'three [SAMPLES OF] mud'. We argued that the main semantic contribution of explicit uses of unit-extracting classifiers is to trigger a context shift, i.e., to make the units for counting accessible. Were this just a freely available pragmatic process for items encoded as mass in the lexicon, we should not expect to see any mass collective artifact nouns, since all would freely accept direct attachment of numerical expressions. This concludes our account of the data from Section 2. However, there are some data that provide major challenges to the account here provided.

\section{Challenges and further extensions}

As has been widely discussed since at least Fargo 1968, the phenomenon of count/mass crosslinguistic variation is not restricted to collective artifacts, but is also commonly exhibited by granular nouns, such as the English rice, lentil(s), pebbles, and gravel. For example, in Bulgarian and many other Slavic languages, the word denoting lentils is lexicalized as mass. This is shown by (44) and (45). Felicity of the singular lešta ('lentil') in (45) indicates that lešta is mass.

(44) Kupi-x 300g jabulk-i / *jabulka. buy-PAST $300 \mathrm{~g}$ apple-PL / apple.SG

'I bought $300 \mathrm{~g}$ of apples / *apple.'

(45) Kupi-x 300g lešta.

buy-PAST $300 \mathrm{~g}$ lentil.SG 
'I bought $300 \mathrm{~g}$ of lentils.'

It is hard to see how we could accommodate these data, within an overlap (disjointness) based theory. If lentil has a non-overlapping IND set, then it surely does so because the individual lentils count as 'one' and do not overlap. However, if only individual lentils count as 'one', then lentil, semantically, looks more like a prototypical count noun and so should display little or no count/mass variation. Our model clearly needs enriching to give it even more explanatory power. We outline one avenue for doing so in Sutton \& Filip 2016a. There, we suggest that a further context index is required that operates in a manner similar to Chierchia's (2010) vagueness based approach. Nonetheless, it remains unclear whether we would be able to replicate the appropriate restrictions on coercion for granular nouns. For example, we see infelicity in examples such as (46):

?? Three rices fell off my fork.

Int: Three grains of rice fell off my fork.

\section{Conclusion}

By including null-counting contexts and counting contexts of utterance in our semantics, we have been able to model two types of data heretofore unexplained in the count/mass literature. We can predict and formally represent count/mass variation amongst collective artifact nouns and we capture the right restrictions to mass-tocount coercion for nouns in this class. The framework given here offers a potential base upon which to build a semantics with even wider coverage in these respects.

\section{References}

Adekomaya, Olufemi A. 2013. Experimental analysis of the effect of magnesium saltwater influx on the behaviour of drilling fluids. Journal of Petroleum Exploration and Production Technology 3. 61-67. doi:10.1007/s13202-012-0046-6.

Chierchia, Gennaro. 1998. Plurality of mass nouns and the notion of "semantic parameter”. In Susan Rothstein (ed.), Events and Grammar: Studies in Linguistics and Philosophy Vol. 7, 53-103. Kluwer. doi:10.1007/978-94-011-3969-4.

Chierchia, Gennaro. 2010. Mass nouns, vagueness and semantic variation. Synthese 174. 99-149. doi:10.1007/s11229-009-9686-6.

Fargo, Nancy L. 1968. Natural language and exact thinking. Annual Report, Neurocommunications Lab., Dept of Psychiatry, John Hopkins University 463476.

Krifka, Manfred. 1989. Nominal reference, temporal constitution and quantification in event semantics. In Renate Bartsch, Johan van Benthem \& Peter 
Counting in context

van Emde Boas (eds.), Semantics and Contextual Expression, 75-115. Foris Publications.

Landman, Fred. 2011. Count nouns - mass nouns - neat nouns - mess nouns. Baltic International Yearbook of Cognition, Logic and Communication 6. 1-67. doi:10.4148/biyclc.v6i0.1579.

Landman, Fred. 2016. Iceberg semantics for count nouns and mass nouns: The evidence from portions. The Baltic International Yearbook of Cognition Logic and Communication 11. (To appear).

Link, Godehard. 1983. The logical analysis of plurals and mass terms: A latticetheoretic approach. In Paul Portner \& Barbera Partee (eds.), Formal Semantics the Essential Readings, 127-147. Blackwell. doi:10.1002/9780470758335.

Pelletier, Francis Jeffry. 1975. Non-singular reference: Some preliminaries. Philosophica 5(4). 451-465. doi:10.1007/978-1-4020-4110-5_1.

Quine, Willard Van Orman. 1960. Word and Object. Cambridge, MA: MIT Press.

Rothstein, Susan. 2010. Counting and the mass/count distinction. Journal of Semantics 27(3). 343-397. doi:10.1093/jos/ffq007.

Rothstein, Susan. 2015. Object mass nouns from a crosslinguistic perspective. Handout. Invited talk at the SFB991 Colloquium, Heinrich Heine University Düsseldorf.

Soja, Nancy, Susan Carey \& Elizabeth Spelke. 1991. Ontological categories guide young children's inductions of word meaning: Object terms and substance terms. Cognition 38. 179-211. doi:10.1016/0010-0277(91)90051-5.

Sutton, Peter R. \& Hana Filip. 2016a. Mass/count variation, a mereological, twodimensional semantics. The Baltic International Yearbook of Cognition Logic and Communication 11. (To appear).

Sutton, Peter R. \& Hana Filip. 2016b. Restrictions on subkind coercion in superordinate object mass nouns. Handout. Presented at Sinn und Bedeutung 2016, University of Edinburgh.

Zucchi, Sandro \& Michael White. 1996. Twigs, sequences and the temporal constitution of predicates. In Teresa Galloway \& Justin Spence (eds.), Semantics and Linguistic Theory (SALT) 6, 223-270. Ithaca, NY: Linguistic Society of America. doi:10.3765/salt.

Zucchi, Sandro \& Michael White. 2001. Twigs, sequences and the temporal constitution of predicates. Linguistics and Philosophy 24(2). 223-270. doi:10.1023/A:1005690022190. 
Peter R. Sutton

Institute for Language and Information Department of General Linguistics

Heinrich Heine University

Düsseldorf

peter.r.sutton@icloud.com

\section{Hana Filip}

Institute for Language and Information Department of General Linguistics Heinrich Heine University Düsseldorf

hana.filip@gmail.com 